\title{
Résultats d'un délai d'application du dégagement mécanique en plantations d'épinettes blanche et noire dans un scénario de reboisement hâtif
}

\author{
par Morgane Urli ${ }^{1,2}$, Martin Barrette ${ }^{1}$, Alain Leduc ${ }^{2}$ et Nelson Thiffault ${ }^{1,3^{*}}$
}

\begin{abstract}
RÉSUMÉ
La maîtrise de la végétation concurrente est essentielle afin que les plantations présentent les rendements escomptés, mais ses effets dépendent de son moment d'application. Ainsi, notre objectif était d'évaluer l'effet, après 15-20 ans, d'un délai d'application du dégagement mécanique par rapport au moment requis selon les procédures opérationnelles au Québec (Canada) pour des plantations de Picea glauca et Picea mariana. Nous avons utilisé trois dispositifs expérimentaux de reboisement hâtif comprenant les traitements suivants : i) témoin, non dégagé; ii) dégagement mécanique l'année requise (Requis); iii) dégagement mécanique avec un délai d'une année par rapport à Requis (Requis+1); et, iv) dégagement mécanique avec un délai de deux années (Requis+2). Nos résultats montrent que dans un scénario de reboisement hâtif comprenant un traitement de nettoiement au stade gaulis, il n'y a pas d'impact significatif sur la croissance à retarder l'application d'un dégagement mécanique jusqu’à deux années après le moment requis. Cette marge opérationnelle devrait être utilisée afin d’assurer la réalisation des dégagements; l’omission du dégagement a un effet négatif marqué sur la production aux échelles de l'arbre et du peuplement. Le dégagement a en effet augmenté la hauteur et le diamètre des arbres plantés ainsi que la surface terrière totale des peuplements.
\end{abstract}

Mots clés : sylviculture, plantation, gestion de la végétation, compétition

\section{ABSTRACT}

Vegetation management is required so that plantations present expected yields, but its impacts depend on the time of application. Our objective was to evaluate the effect, after 15-20 years, of delaying the application of a mechanical release treatment in Picea glauca and Picea mariana plantations, relative to the timing prescribed based on operational guidelines in Quebec (Canada). We used three experimental sites established following an early planting scenario that comprised the following treatments: i) control, no release; ii) mechanical release applied during the required year with motor-manual brushsaws; iii) mechanical release applied one year after the required year; and, iv) mechanical release two years after the required year. Our results show that, under an early planting scenario that includes a cleaning treatment at the sapling stage, there is no significant impact on plantation growth to delay the mechanical release for up to two years compared to the required year. This operational window should be used to ensure that vegetation management treatments are performed; omitting release treatments at the seedling stage has a marked negative effect on tree and stand productivity. Mechanical release indeed increased tree height, diameter, and stand total basal area.

Keywords: silviculture, plantation, vegetation management, competition

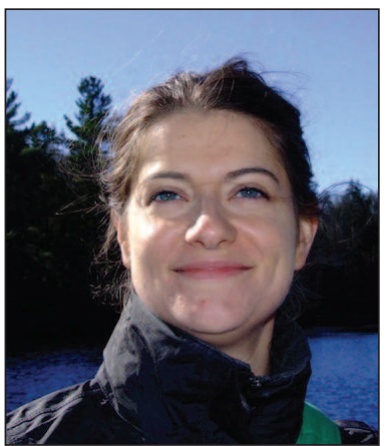

Morgane Urli



Martin Barrette



Alain Leduc

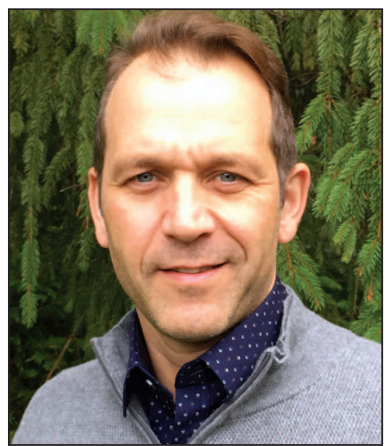

Nelson Thiffault

\footnotetext{
${ }^{1}$ Direction de la recherche forestière, Ministère des Forêts, de la Faune et des Parcs du Québec, 2700 Einstein, Québec, QC G1P 3W8, Canada ${ }^{2}$ Centre détude de la forêt, Université du Québec à Montréal, Case postale 8888, succursale Centre-ville, Montréal, QC H3C 3P8, Canada ${ }^{3}$ Centre détude de la forêt, Faculté de foresterie, de géographie et de géomatique, Université Laval, Québec, QC G1V 0A6, Canada *Auteur de correspondance. Adresse actuelle : Centre canadien sur la fibre de bois, Service canadien des forêts, Ressources naturelles Canada, 1055, rue du P.E.P.S., C.P. 10380, succ. Sainte-Foy, Québec, QC G1V 4C7 (nelson.thiffault@canada.ca)
} 


\section{Introduction}

La productivité des plantations est un enjeu incontournable pour atteindre un aménagement durable des forêts (Park and Wilson 2007). En effet, les plantations forestières ont un rendement ligneux élevé qui dépasse généralement celui des forêts naturelles (Prégent et al. 2010; Bureau du forestier en chef 2013). Ceci permet, notamment, de les utiliser pour augmenter la productivité forestière sur des superficies dédiées, afin de rencontrer les impératifs économiques de l’aménagement forestier durable, et de supporter la mise en place d'aires protégées (Ward and Erdle 2015). Dans ce contexte, la foresterie de plantation est appelée à jouer un rôle de plus en plus important à léchelle mondiale (Paquette and Messier 2010). Au Canada seulement, plus de 574 millions de plants ont été mis en terre en 2015, sur plus de 413000 ha (Base de données nationale sur les forêts 2017). Au Québec, ce sont près de 114 millions de plants qui ont été mis en terre cette même année, sur 68000 ha (Salmon 2016).

Toutefois, le succès détablissement et la productivité des plantations sont variables et soumis à l'influence de nombreux facteurs, dont la composition et l'abondance de la végétation concurrente. Suite à une coupe forestière, les parterres sont rapidement dominés par des espèces de début de succession qui, le plus souvent, exercent une forte compétition pour la lumière, leau et les éléments nutritifs à l'endroit des arbres plantés (Wagner and Robinson 2006). Alors que les plus fortes pertes de croissance sont occasionnées par les premiers compétiteurs feuillus (e.g., érable à épis, Acer spicatum Lam.; bouleau blanc, Betula papyrifera Marsh.; cerisier de Pennsylvanie, Prunus pensylvanica L.f.) (Jobidon 2000), les herbacées et les arbustes ont également un impact significatif sur la survie et la croissance des arbres plantés (Bell et al. 2000; Hoepting et al. 2011). La présence de végétation concurrente réduit également la biomasse foliaire des plants mis en terre (Will et al. 2006), ce qui diminue leur capacité à capter la lumière et réduit ainsi leur potentiel compétitif (Jobidon et al. 1998). Ainsi, les pratiques sylvicoles associées à la gestion de la compétition ont un effet décisif sur le devenir des plantations (Wagner et al. 2006).

La maitrise de la végétation forestière est traditionnellement réalisée à laide de phytocides chimiques, tel le glyphosate. Le dégagement chimique est efficace pour diminuer la compétition par les espèces concurrentes, ce qui stimule la croissance des essences désirées (e.g., Pitt et al. 2000). Cependant, son utilisation soulève des enjeux d'acceptabilité sociale (Wyatt et al. 2011) qui ont mené certaines juridictions à abandonner son utilisation sur les terres publiques, ou encore, à cesser de la subventionner. Par exemple, au Québec, la gestion de la végétation concurrente sans phytocides repose sur le dégagement mécanique à l'aide de débroussailleuses motorisées (Thiffault and Roy 2011). Cette pratique vise à sassurer que les essences d'intérêt dominent létage dominant des peuplements traités, que la production à l'hectare de ces essences est augmentée, et que les plantations peuvent ainsi être soumises plus rapidement à un traitement déclaircie commerciale (Gravel et al. 2014). Le dégagement mécanique a prouvé son efficacité lorsqu'il est combiné au reboisement hâtif, i.e., une mise en terre des plants l'année suivant la coupe (Thiffault et al. 2014a). Ainsi, un délai dans l'application du dégagement mécanique pourrait compromettre la productivité de la plantation dans un contexte de gestion de la végétation concurrente sans phytocides. Or, les contraintes liées à la planification forestière, aux budgets ou à la disponibilité d'une main-d’ouvre qualifiée rendent parfois difficile, voire impossible, l'application des traitements de dégagement aux moments recommandés. Au Québec, les critères d’application du dégagement mécanique sont basés, notamment, sur la proportion de la pleine lumière reçue par les semis (Jobidon 1992, 1994), selon des seuils déterminés en fonction de l'autoécologie des essences plantées (e.g., $60 \%$ pour Picea glauca (Moench) Voss et P. mariana (Mill.) BSP; Gravel et al. 2014). Il importe ainsi de préciser comment, dans un scénario de reboisement hâtif qui comprend également un traitement de nettoiement au stade gaulis, le délai dans l'application du dégagement mécanique influence la croissance subséquente des arbres plantés.

Nos objectifs étaient donc d'évaluer, à léchelle de l’arbre et du peuplement, l'effet d'un délai d'un ou deux ans dans l'application d'un traitement de dégagement mécanique par rapport au moment requis selon les procédures opérationnelles en vigueur au Québec, pour des plantations d'épinette blanche et noire. Notre première prédiction était qu'un délai dans l’application du dégagement mécanique réduirait la dimension des tiges d'épinettes blanche et noire et le rendement des peuplements. Notre seconde prédiction était que le dégagement de la compétition en bas âge influencerait la réponse des arbres d'intérêt à la compétition au stade juvénile. Pour vérifier la première prédiction, nous avons évalué l'effet d'un délai dans lapplication du dégagement mécanique sur la survie, la hauteur et le diamètre d’épinettes blanche et noire en monoculture, entre 15 et 20 ans après leur mise en terre, et nous avons déterminé comment ces effets se manifestent à léchelle du peuplement au regard de la surface terrière. Pour vérifier la seconde prédiction, nous avons évalué comment les traitements sylvicoles effectués en bas âge influent sur la réponse des arbres à la compétition à moyen terme, en explorant la relation entre le scénario de dégagement et un indice de compétition dépendant de la distance des plus proches voisins.

\section{Matériels et Méthodes \\ Stations d'étude}

Nous avons évalué les effets à moyen terme (15-20 ans) du délai d'application d'un dégagement mécanique à laide de données issues de trois dispositifs expérimentaux tirés d'un réseau plus vaste qui couvre le Québec méridional (Thiffault et al. 2014b). Les trois dispositifs, ci-après identifiés "Owen ", "Sainte-Perpétue " et "Caseault ", sont localisés dans les domaines bioclimatiques de la sapinière (Abies balsamea (L.) Mill.) à bouleau jaune (Betula alleghaniensis Britt.) du Québec (Saucier et al. 2009), (Owen et Sainte-Perpétue) ou de la sapinière à bouleau blanc (Caseault; Fig. 1). Le climat est subhumide continental, avec des précipitations annuelles variant de 1000 à $1100 \mathrm{~mm}$, et une température annuelle moyenne de $2,5^{\circ} \mathrm{C}$ (Robitaille et Saucier 1998). Les sols sont des podzols (Soil Classification Working Group 1998) à texture de loam ou loam sableux, formés à partir de dépôts de tills indifférenciés ou d'altération. Les peuplements dorigine, résineux ou mélangés à dominance résineuse, ont été récoltés en 1994 (Caseault) ou 1995 (Owen et Sainte-Perpétue) par coupe avec protection de la régénération et des sols. En raison d’une régé- 
(a)

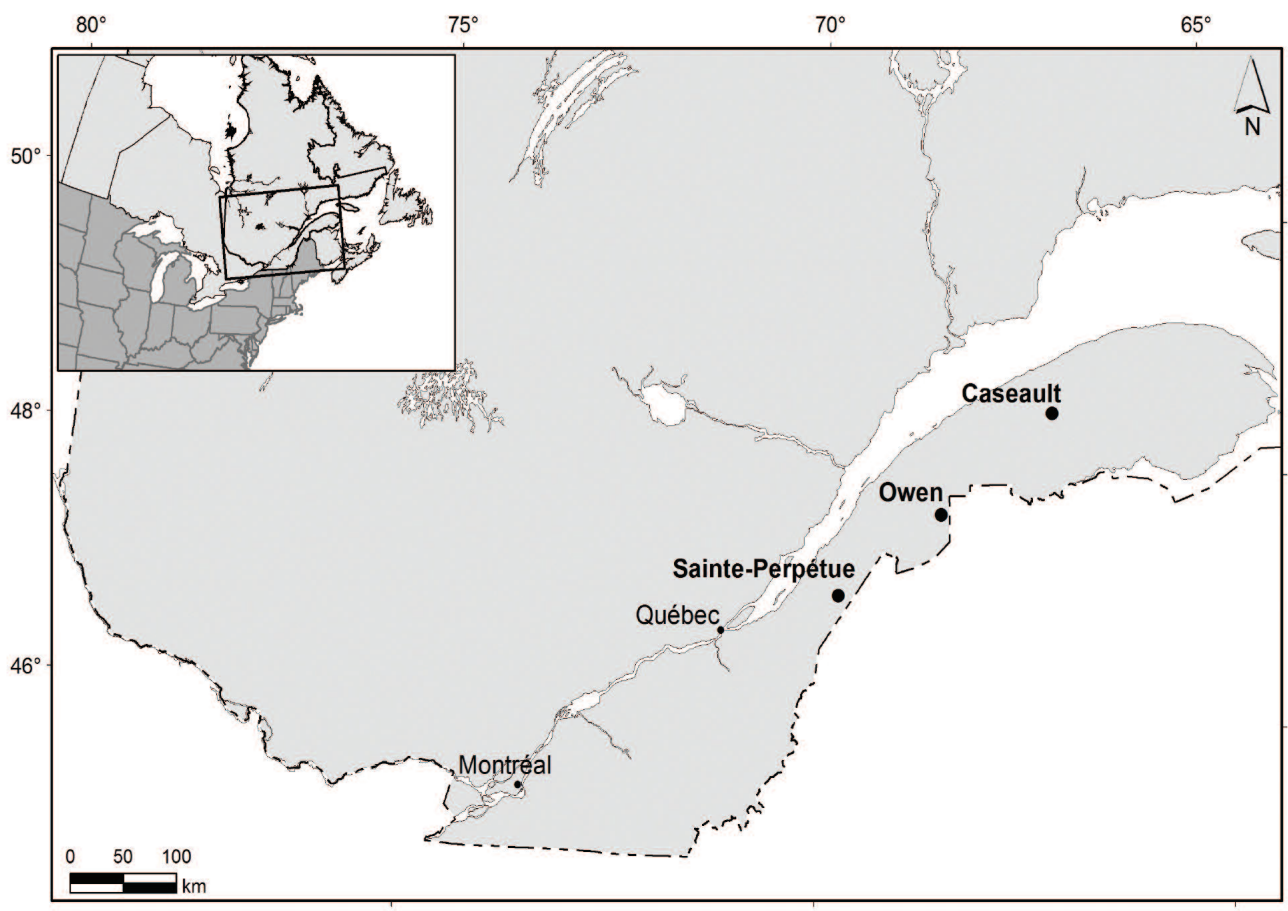

(b)

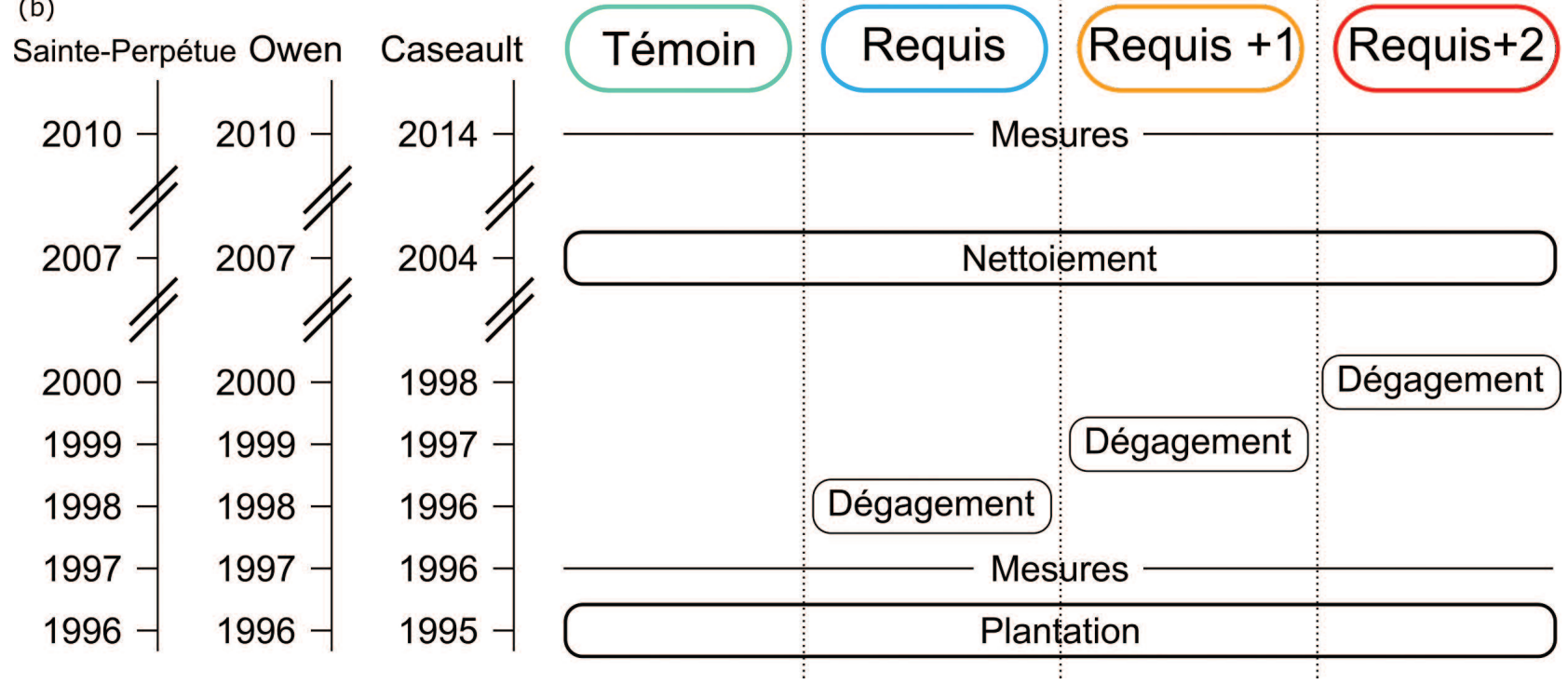

Fig. 1. Localisation des dispositifs expérimentaux (a). Chronologie de l'application des traitements sylvicoles et des campagnes de mesures (b).

nération insuffisante, ils ont été reboisés le printemps suivant la coupe (juin 1995 pour Caseault; juin 1996 pour Owen et Sainte-Perpétue). Dans tous les cas, le reboisement a été précédé d'une mise en andains des débris de coupe afin de permettre l'accès sécuritaire aux sites pour les travailleurs forestiers. Les sites ont été reboisés de manière opérationnelle avec des plants dépinette blanche (Owen et Sainte-Perpétue) ou noire (Caseault) à une densité cible de 2000 plants/ha. Les plants ont été produits en récipients d'un volume de carotte racinaire variant de 110 à $350 \mathrm{~cm}^{3}$, choisis selon les caractéristiques des stations (Thiffault and Roy 2011). 

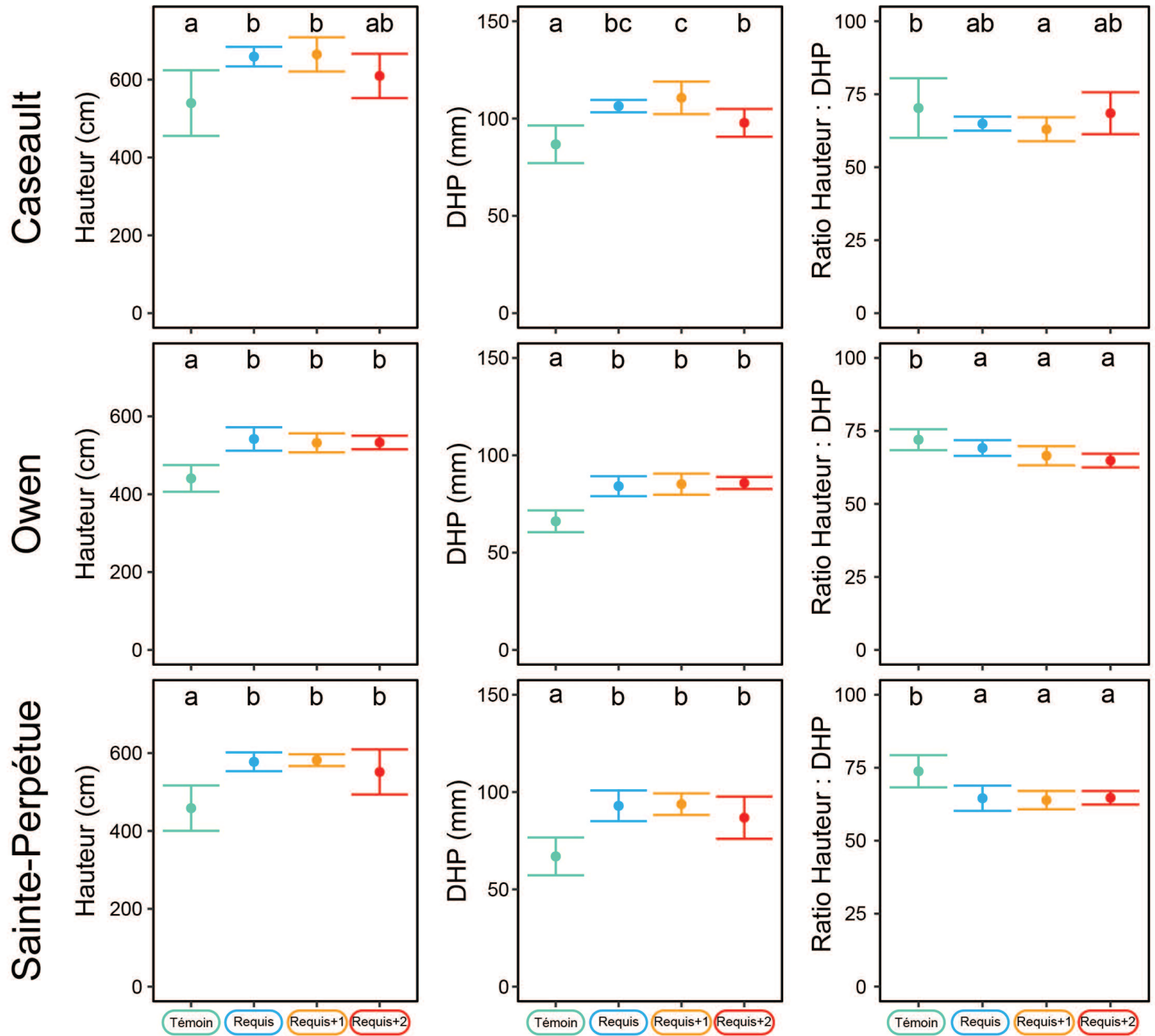

Fig. 2. Valeurs de la hauteur $(\mathrm{cm})$, du DHP $(\mathrm{mm})$ et du ratio Hauteur:DHP des épinettes plantées dans les peuplements non soumis à un dégagement mécanique (Témoin) et dans ceux soumis à un dégagement l'année requise (Requis], une année après l'année requise (Requis+1) ou deux années après l'année requise (Requis+2) pour les trois sites d'étude : plantation d'épinette noire (Caseault) et plantations d'épinette blanche (Owen et Sainte-Perpétue). Les données présentées sont les moyennes \pm l'écart-type (n $\leq 7$ blocs). Les moyennes avec les mêmes lettres ne sont pas significativement différentes $(\alpha=0,05)$.

\section{Dispositifs expérimentaux}

Nous avons établi chacune des plantations expérimentales selon un design en blocs complets entièrement aléatoires. Ainsi, à chacun des sites, nous avons délimité de 5 à 7 blocs expérimentaux pendant la deuxième saison de croissance depuis la mise en terre des plants. Chacun des blocs mesurait $34 \times 28 \mathrm{~m}$; nous les avons divisés en quatre parcelles (unités expérimentales) de $7 \times 28 \mathrm{~m}$ avec des zones tampons de $2 \mathrm{~m}$ entre chacune d'elle. Nous avons attribué, à chaque parcelle, l'un des quatre traitements suivant au hasard (Fig. 1) : i) témoin, non dégagé; ii) dégagement mécanique réalisé en juillet-août de l'année requise selon les normes opération- nelles en vigueur au Québec (Gravel et al. 2014), soit entre 1996 et 1998, selon le site (Requis); iii) dégagement mécanique réalisé avec un délai d'une année par rapport à Requis, soit entre 1997 et 1999, selon le site (Requis +1); et iv) dégagement mécanique réalisé avec un délai de deux années, soit entre 1998 et 2000, selon le site (Requis +2). Neuf ans (Caseault) ou 11 ans (Owen et Sainte-Perpétue) après la mise en terre des plants, lensemble des dispositifs (y compris les parcelles témoins) a été soumis à un traitement de nettoiement mécanique (sensu Thiffault et Hébert 2013) à l'aide de débroussailleuses motorisées (Fig. 1). Pour chacun des dispositifs, l'année du nettoiement a été déterminée sur la base de 
Tableau 1. Résultats des ANOVAs testant l'effet des scénarios sylvicoles sur la hauteur [cm], le diamètre à hauteur de poitrine (DHP, mm) et le ratio Hauteur/DHP des épinettes plantées pour les trois sites d'étude : plantation d'épinette noire (Caseault) et plantations d'épinette blanche [Owen et Sainte-Perpétue]. Les valeurs en gras sont significatives au seuil $\boldsymbol{\alpha}=0,05$.

\begin{tabular}{|c|c|c|c|c|c|c|c|c|c|}
\hline \multirow[b]{2}{*}{ ANOVA } & \multicolumn{3}{|c|}{ Caseault } & \multicolumn{3}{|c|}{ Owen } & \multicolumn{3}{|c|}{ Sainte-Perpétue } \\
\hline & $D D L$ & $F$ & $P$ & $D D L$ & $F$ & $P$ & $D D L$ & $F$ & $P$ \\
\hline Hauteur & $3,14,95$ & 1222 & $<0,001$ & $3,16,55$ & 23,37 & $<0,001$ & $3,9,84$ & 17,55 & $<0,001$ \\
\hline DHP & $3,14,81$ & 16,46 & $<0,001$ & $3,15,93$ & 33,34 & $<0,001$ & $3,9,29$ & 28,15 & $<0,001$ \\
\hline $\begin{array}{l}\text { Ratio } \\
\text { Hauteur: } \\
\text { DHP }\end{array}$ & $3,9,33$ & 7,66 & 0,007 & $3,16,59$ & 15,58 & $<0,001$ & $3,12,73$ & 13,76 & $<0,001$ \\
\hline
\end{tabular}

DDL : degrés de liberté au numérateur, degrés de liberté au dénominateur

labondance, de la nature et de la hauteur de la végétation concurrente observée dans les parcelles soumises au traitement Requis, et ce, afin de représenter le scénario sylvicole opérationnel qui aurait été appliqué dans ces mêmes conditions (Gravel et al. 2014). Pour un site donné, l'utilisation d'une année commune pour réaliser un nettoiement dans toutes les parcelles, sans égard à l'année du dégagement, a résulté en quatre scénarios sylvicoles qui diffèrent quant à lâge de la plantation au moment où le dégagement a été réalisé, et au nombre d’années entre le dégagement et le nettoiement (Fig. 1). Chacun des plants a été identifié avec une tige métallique et un numéro unique afin de faire le suivi de sa survie et de sa croissance à long terme. Le premier mesurage (hauteur en cm, et diamètre au niveau du sol en $\mathrm{mm}$ ) a eu lieu deux ans après la mise en terre. Au total, les dispositifs de Caseault, Owen et Sainte-Perpétue comptaient respectivement 1078, 1522 et 764 plants numérotés, répartis également dans les quatre traitements.

\section{Mesures des plants et des gaules de conifères}

Les plants numérotés toujours vivants ont été mesurés 15 (Owen et Sainte-Perpétue) ou 20 ans (Caseault ; Fig. 1) après la mise en terre. Nous avons noté la hauteur $(\mathrm{cm})$ et le diamètre à hauteur de poitrine des tiges (DHP en $\mathrm{mm}$, mesuré à $1,30 \mathrm{~m}$ ). De même, la hauteur et le DHP des tiges de conifères issues de régénération naturelle et mesurant au moins $1,30 \mathrm{~m}$ de hauteur et au moins $11 \mathrm{~mm}$ de DHP (ci-après désignées comme « recrues ») ont été mesurés.

\section{Mesures des plus proches voisins et des feuillus}

Sur chaque site, dans chaque unité expérimentale, nous avons délimité deux placettes semi-circulaires de 5,64 $\mathrm{m}$ de rayon, localisées approximativement au premier et deuxième tiers des parcelles expérimentales, de part et d'autre de la parcelle rectangulaire. Pour une des deux placettes semi-circulaires $\left(50 \mathrm{~m}^{2}\right)$, nous avons identifié lespèce, mesuré la hauteur, ainsi que la distance des quatre plus proches voisins des arbres plantés. Nous avons mesuré la hauteur et le DHP de toutes les tiges feuillues de plus de 1,30 $\mathrm{m}$ de hauteur dans les deux placettes semi-circulaires (totalisant $100 \mathrm{~m}^{2}$ ).

\section{Analyses statistiques}

L’ensemble des analyses a été réalisé pour chaque site séparément en raison de leur spécificité en regard des essences plantées, de l'année de la plantation et du volume initial de la carotte racinaire de plants. Les analyses ont été effectuées à l'aide d'analyses de variance (ANOVA) pour évaluer les effets des quatre scénarios sylvicoles sur les variables étudiées. Nous avons utilisé des modèles linéaires mixtes (Eq. [1]) pour tester l'effet des scénarios sylvicoles sur la hauteur, le DHP et le ratio Hauteur:DHP à la 15ème (Owen et SaintePerpétue) ou 20ème (Caseault) année après la mise en terre des plants, ces variables ayant été mesurées à léchelle de l’arbre.

$$
\text { [1] } Y_{i j k}=\mu+B_{\mathrm{i}}+S_{\mathrm{j}}+(B \times S)_{\mathrm{ij}}+\varepsilon_{k(i j)}
$$

où $Y_{i j k}$ représente la valeur de la variable concernée, $\mu$ correspond à la moyenne globale, $B_{i}$ concerne l'effet aléatoire du bloc $i(i=1$ à 5,6 ou 7$)$ avec $B_{i} \sim N\left(0, \sigma_{B}^{2}\right)$ et $S_{j}$ représente l'effet fixe du scénario sylvicole $j(j=1$ à 4$)$. Le terme $(B \times S)_{i j}$ est l'interaction entre le bloc $i$ et le scénario sylvicole $j$, définissant les unités expérimentales et correspondant à lerreur expérimentale $\left((B \times S)_{i j} \sim N\left(0, \sigma_{B \times S}^{2}\right)\right)$, alors que $\varepsilon_{k(i j)}$ représente l'erreur résiduelle, correspondant aux arbres à 'intérieur de chacune des unités expérimentales $\left(\varepsilon_{k(i j)} \sim N\left(0, \sigma_{\varepsilon}^{2}\right)\right)$.

Nous avons également utilisé des modèles linéaires mixtes pour évaluer l'effet des scénarios sylvicoles sur la survie des plants mis en terre, les valeurs de la surface terrière des épinettes plantées, des conifères et des feuillus régénérés naturellement et leur proportion en surface terrière dans le peuplement, ainsi que sur la surface terrière totale, mais en supprimant l'interaction entre les blocs et les scénario sylvicoles, ces variables ayant été calculées à l'échelle de l'unité expérimentale (Eq. [2]) :

$$
\text { [2] } Y_{i j}=\mu+B_{i}+S_{j}+\varepsilon_{i j}
$$

où la définition des différents termes est identique à celle de l'équation [1], à l'exception de l'erreur résiduelle $\varepsilon_{i j}$ qui correspond ici aux unités expérimentales, soit l'interaction entre les blocs et les scénarios sylvicoles.

Des tests post hoc de Tuckey ont été effectués pour tester les différences entre scénarios sylvicoles lorsque la valeur $F$ de l'ANOVA était significative à $\alpha=0,05$.

Nous avons utilisé les données relatives aux quatre plus proches voisins des épinettes qui se trouvaient dans les placettes de $50 \mathrm{~m}^{2}$ pour calculer la hauteur angulaire, un indice de compétition dépendant de la distance (Biging et Dobbertin 1992). Pour chaque arbre planté, nous avons calculé une hauteur angulaire moyenne des quatre voisins. Nous avons ensuite réalisé des régressions linéaires mixtes selon le modèle suivant :

[3] $Y_{i j k}=\mu+B_{i}+S_{j}+H_{k(i j)}+(B \times S)_{i j}+(S \times H)_{k(i j)}+\varepsilon_{k(i j)}$ 


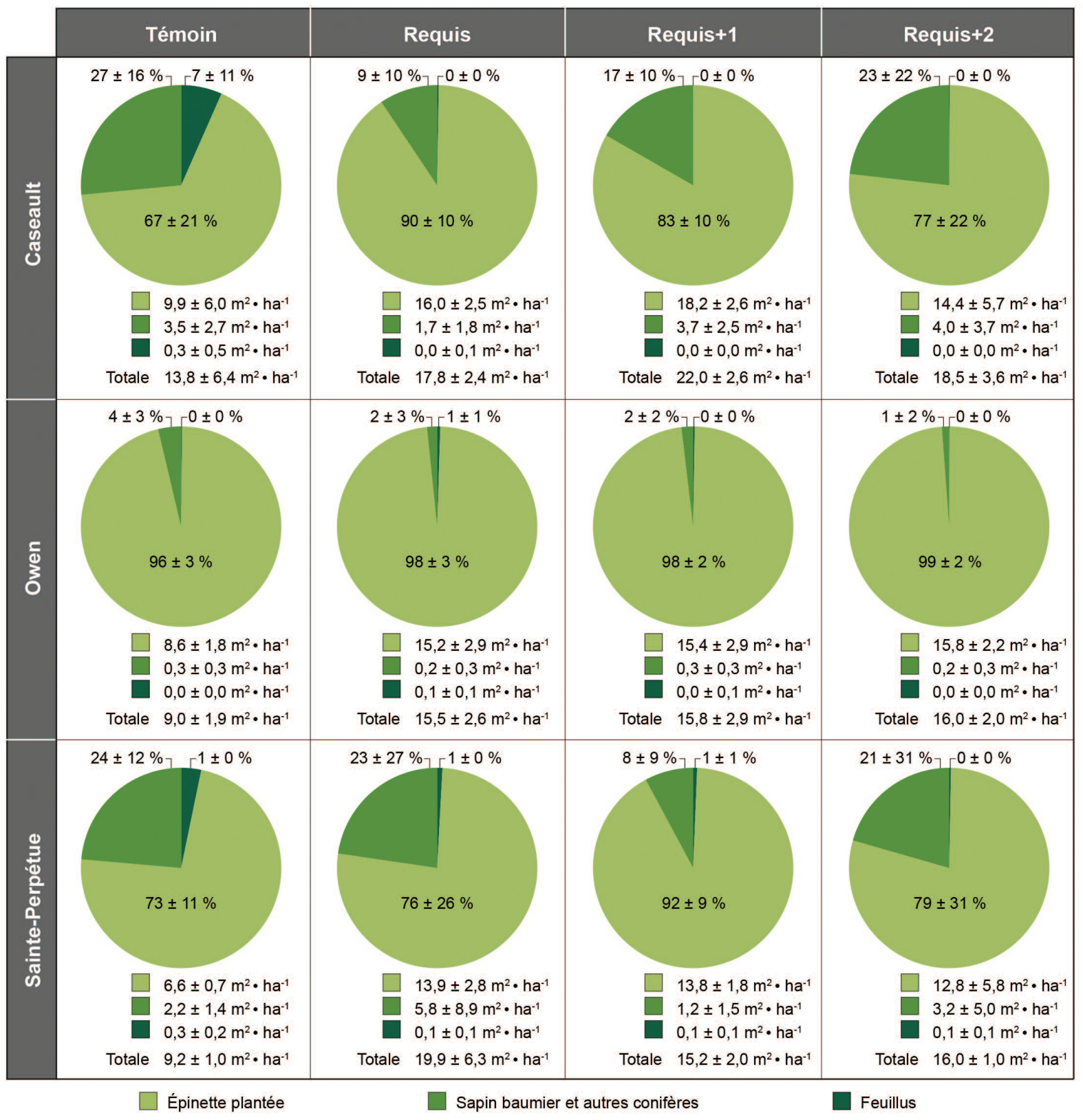

Fig. 3. Proportions (dans les graphiques circulaires) et valeurs absolues (sous les graphiques circulaires) de la surface terrière occupée par les différents groupes d'essences dans les peuplements non soumis à un dégagement mécanique (Témoin) et dans ceux soumis à un dégagement l'année requise (Requis), un an après l'année requise (Requis+1) ou deux ans après l'année requise (Requis+2) pour les trois sites d'étude : plantation d'épinette noire (Caseault) et plantations d'épinette blanche (Owen et Sainte-Perpétue). Les données présentées sont les moyennes \pm l'écart-type ( $\mathrm{n} \leq 7$ blocs).

où $Y_{i j k}$ représente le DHP de l'arbre et les termes $\mu, B_{i}, S_{j}$, $(B \times S)_{i j}$ et $\varepsilon_{(k(i j)}$ ont la même définition que dans l'équation [1]. Le terme $H_{k(i j)}$ représente l'effet fixe de la hauteur angulaire moyenne de larbre $k$ situé dans le bloc i ayant reçu le scénario sylvicole $j(k=1$ à $n)$ alors que $(S \times H)_{k(i j)}$ correspond à la pente de la relation entre le DHP et la hauteur angulaire moyenne pour le scénario sylvicole $j$.

L'interaction hauteur angulaire $\mathrm{x}$ scénario sylvicole nétant pas significative pour Owen et Sainte-Perpétue $\left(F_{3}\right.$, $313,6=1234, P=0.297$ et $F_{3,152,3}=1929, P=0.127$, respective- ment) et marginalement significative pour Caseault $\left(F_{3,163,0}=2627, P=0.052\right)$, nous avons considéré une pente commune pour tous les scénarios sylvicoles en supprimant l'interaction fixe des modèles mixtes.

Nous avons vérifié la normalité et l'homoscédasticité des données à laide de visualisations graphiques (histogrammes des données, diagrammes quantile-quantile, diagrammes des résidus en fonction des valeurs estimées) et de tests de Shapiro. Lorsque ces conditions nétaient pas rencontrées, nous avons transformé les données par la racine carrée ou le 
Tableau 2. Résultats des ANOVAs testant l'effet des scénarios sylvicoles sur la surface terrière $\left(\mathrm{m}^{2}\right.$.ha $\left.\mathrm{h}^{-1}\right]$ des épinettes plantées, des conifères et des feuillus régénérés naturellement, sur la surface terrière totale du peuplement $\left[\mathrm{m}^{2} \cdot \mathrm{ha}^{-1}\right]$ et sur leur proportion dans le peuplement [\%] pour les trois sites d'étude : plantation d'épinette noire [Caseault] et plantations d'épinette blanche [Owen et Sainte-Perpétue]. Les valeurs en gras sont significatives au seuil $\boldsymbol{\alpha}=0,05$.

\begin{tabular}{|c|c|c|c|c|c|c|c|c|c|}
\hline \multirow[b]{2}{*}{ ANOVA } & \multicolumn{3}{|c|}{ Caseault } & \multicolumn{3}{|c|}{ Owen } & \multicolumn{3}{|c|}{ Sainte-Perpétue } \\
\hline & $D D L$ & $F$ & $P$ & $D D L$ & $F$ & $P$ & $D D L$ & $F$ & $P$ \\
\hline \multicolumn{10}{|c|}{ Épinettes plantées } \\
\hline Surface terrière & 3,15 & 9,0656 & 0,001 & 3,18 & 26,168 & $<0,001$ & 3,12 & 5,8865 & 0,010 \\
\hline Proportion & 3,15 & 4,709 & 0,016 & 3,18 & 1,474 & 0,255 & 3,12 & 0,87755 & 0,480 \\
\hline \multicolumn{10}{|l|}{ Autres conifères } \\
\hline Surface terrière & 3,15 & 1,375 & 0,289 & 3,24 & 0,490 & 0,692 & 3,12 & 1,2151 & 0,347 \\
\hline Proportion & 3,15 & 4,706 & 0,016 & 3,24 & 1,598 & 0,216 & 3,12 & 1,7781 & 0,205 \\
\hline \multicolumn{10}{|l|}{ Feuillus } \\
\hline Surface terrière & 3,15 & 2,248 & 0,125 & 3,24 & 0,837 & 0,491 & 3,16 & 4,5989 & 0,017 \\
\hline Proportion & 3,20 & 1,947 & 0,155 & 3,24 & 0,953 & 0,431 & 3,12 & 7,2622 & 0,005 \\
\hline \multicolumn{10}{|l|}{ Totale } \\
\hline Surface terrière & 3,15 & 6,480 & 0,005 & 3,18 & 25,151 & $<0,001$ & 3,16 & 11,336 & $<0,001$ \\
\hline
\end{tabular}

DDL : degrés de liberté au numérateur, degrés de liberté au dénominateur

logarithme naturel. Ces analyses ont été réalisées sur $\mathrm{R}$ version 3.4.0 (R Core Team 2012) à l'aide de la librairie lmerTest (Kuznetsova et al. 2016). Pour toutes les analyses, les degrés de liberté associés aux dénominateurs pour les tests des effets fixes ont été calculés avec la méthode de Satterthwaite; les facteurs à effets aléatoires possédaient peu de modalités et la distribution de leurs estimateurs est mieux approximée dans ce cas par la distribution khi-deux que par la loi normale (Littell et al. 2006).

\section{Résultats}

Survie

Nous naavons observé aucune différence de survie entre les scénarios sylvicoles, et ceci, pour tous les sites (Caseault: $F_{3,15}=2,25, P=0,125$; Owen : $F_{3,18}=1,62, P=0,220$ et Sainte-Perpétue : $\left.F_{3,12}=0,57, P=0,644\right)$. La survie des épinettes plantées était supérieure à $65 \%$ à Caseault (après 20 ans) et à $75 \%$ à Owen et Sainte-Perpétue (après 15 ans).

\section{Taille des arbres et surface terrière}

Le délai dans l'application du dégagement mécanique n’a pas influencé la hauteur, le DHP et le ratio Hauteur: DHP des épinettes noire ou blanche (Fig. 2). Toutefois, le dégagement mécanique a résulté en une augmentation significative de la hauteur et du DHP de lépinette blanche par rapport au témoin, de même quen une diminution du ratio Hauteur: DHP (Fig. 2, Tableau 1). Nous avons observé une tendance similaire, mais moins marquée, pour l'épinette noire (Fig. 2, Tableau 1); la différence de hauteur et du ratio Hauteur: DHP entre les parcelles témoins et celles soumises à un dégagement mécanique nétait pas toujours significative (Fig. 2).

La surface terrière des épinettes noires plantées et sa proportion dans le peuplement ne présentaient pas de différence significative entre le délai d'application du dégage- ment mécanique mais étaient supérieures dans les parcelles soumises à un dégagement mécanique par rapport aux parcelles témoins (Fig. 3, Tableau 2, Tableau A1). Bien que les valeurs absolues des surfaces terrières des conifères régénérés naturellement à Caseault ne présentaient pas de différence significative selon les scénarios sylvicoles, leur proportion dans le peuplement était significativement inférieure dans les parcelles témoins que dans les parcelles ayant été dégagées l'année requise (Fig. 3, Tableau 2, Tableau A1). Malgré une différence de $7 \%$ et de $0,3 \mathrm{~m}^{2}$.ha ${ }^{-1}$ des surfaces terrières relative et absolue des feuillus, respectivement, entre les parcelles témoins et les parcelles dégagées, l'effet des traitements s'est avéré ne pas être significatif sur ces variables à Caseault (Fig. 3, Tableau 2).

La surface terrière des épinettes blanches plantées et sa proportion dans le peuplement ne présentaient pas de différence significative entre le délai d’application du dégagement mécanique. Les parcelles dégagées mécaniquement avaient toutefois une surface terrière d'épinette blanche plantée deux fois plus élevée que les parcelles témoins (Fig. 3, Tableau 2, Tableau A1). La surface terrière des conifères non plantés et sa proportion dans le peuplement nétaient pas significativement différentes entre les scénarios sylvicoles (Fig. 3, Tableau 2). À Sainte-Perpétue, le dégagement mécanique a significativement diminué la surface terrière des feuillus et sa proportion dans le peuplement, représentés principalement par le sureau pubescent (Sambucus racemosa subsp. pubens (Michaux) Hultén) (Fig. 3, Tableau 2).

Le délai dans l'année d'application du dégagement mécanique n’a pas eu d'effet sur la surface terrière totale (Fig. 3 , Tableau 2). Le dégagement mécanique a conduit à une augmentation significative de la surface terrière totale des peuplements, par rapport au témoin (Fig. 3, Tableau 2). Toutefois, la surface terrière des conifères établis naturellement était principalement représentée par des recrues de 

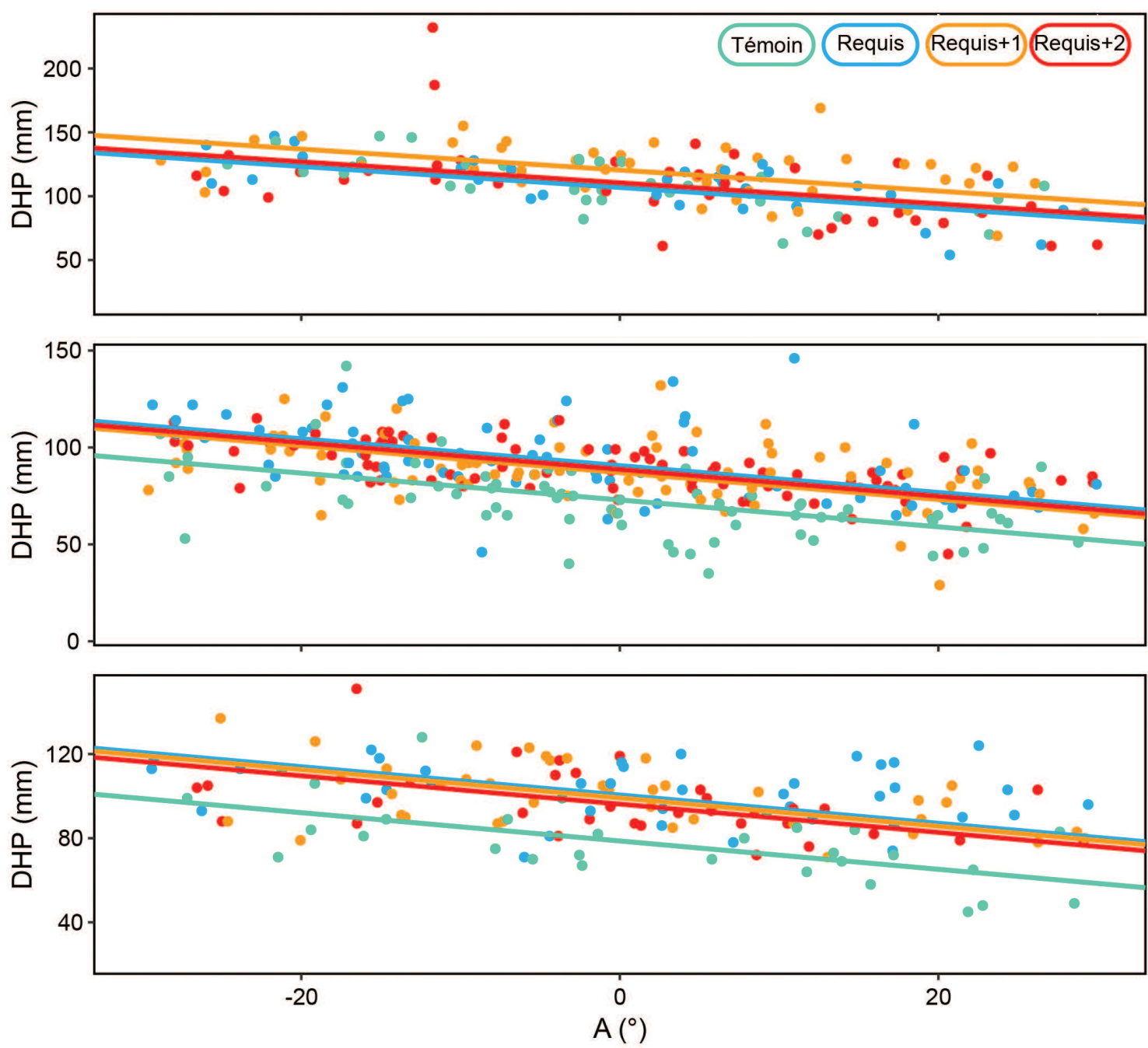

Fig. 4. Réponse du diamètre à hauteur de poitrine $(\mathrm{DHP}, \mathrm{mm})$ à l'augmentation de la hauteur angulaire (indice de compétition, $\left.A,{ }^{\circ}\right)$ dans les peuplements non soumis à un dégagement mécanique (Témoin) et dans ceux soumis à un dégagement l'année requise (Requis), un an après l'année requise (Requis+1) ou deux ans après l'année requise (Requis+2) pour les trois sites d'étude : plantation d'épinette noire (Caseault) et plantations d'épinette blanche (Owen et Sainte-Perpétue). haque point représente le DHP de l'arbre planté en fonction de la moyenne des valeurs de la hauteur angulaire entre l'arbre planté et ses quatre voisins les plus proches. Une valeur de $A<0$ signifie que l'arbre planté est plus grand que la moyenne de ses quatre plus proches voisins. Les lignes représentent la régression linéaire du $D H P$ en fonction de la hauteur angulaire selon les équations suivantes : $D H P=-0,822 A+109,376 ; \quad D H P=-0,822 A$ $+106,836 ; D H P=-0,822 A+120,445 ; D H P=-0,822 A+110,484$ pour les scénarios sylvicoles Témoin, Requis, Requis+1 et Requis+2, respectivement de Caseault; $D H P=-0,693 A+72,904 ; D H P=-0,693 A+90,624 ; D H P=-0,693 A+86,993 ; D H P=$ $-0,693 A+88,682$ pour les scénarios sylvicoles Témoin, Requis, Requis+1 et Requis+2, respectivement de Owen; et $D H P=-0,673 A$ $+78,677 ; \quad D H P=-0,673 A+100,537 ; D H P=-0,673 A+99,135 ; D H P=-0,673 A+96,223$ pour les scénarios sylvicoles Témoin, Requis, Requis+1 et Requis+2, respectivement de Sainte-Perpétue.

sapin baumier et d'épinette noire dans la plantation d'épinette noire (Caseault), et de sapin baumier et d'épinette blanche dans les plantations d'épinette blanche (Owen et Sainte-Perpétue).

\section{Compétition}

À chacun des sites, le DHP des épinettes plantées a significativement diminué avec l'augmentation de la hauteur angulaire moyenne des plus proches voisins (Caseault : $F_{1,165,5}=$
121,52, $P<0,001$; Owen : $F_{1,316,4}=311,71, P<0,001$; Sainte-Perpétue : $F_{1,150,0}=134,10, P<0,001$, Fig. 4 ), et ce, selon un taux similaire pour chacun des scénarios sylvicoles (cf. Matériels et Méthodes). L’ordonnée à lorigine des régressions linéaires nétait pas significativement différente entre les scénarios sylvicoles pour les arbres de Caseault $\left(F_{3,13,8}=\right.$ $2,86, P=0.075$, Fig. 4). Cependant, les parcelles témoins situées à Owen et Sainte-Perpétue présentaient des ordonnées à lorigine inférieures aux parcelles ayant été soumises à 
un traitement de dégagement (Owen : $F_{3,21,0}=14,61, P<$ 0,001; Sainte-Perpétue : $F_{3,7,1}=16,97, P=0,001$; Fig. 4); les ordonnées à l'origine des traitements Requis, Requis+1 et Requis +2 étaient équivalentes $(P \geq 0,05)$.

\section{Discussion}

Contrairement à nos prédictions, un délai d'un ou deux ans dans l'application du dégagement mécanique par rapport au moment requis selon les procédures opérationnelles en vigueur au Québec n'a pas réduit la productivité à moyen terme de plantations d'épinette blanche ou noire. Notre étude démontre que, dans un scénario de plantation qui comprend un reboisement hâtif suivi d'un dégagement et d'un nettoiement au stade gaulis, il y a peu d'impacts à retarder le dégagement mécanique du stade semis jusqu'à deux saisons après le moment requis pour des plantations établies sur stations riche de la sapinière. Le reboisement hâtif, associé à l'utilisation de plants d'une taille initiale adaptée aux conditions de stations, crée une fenêtre d'intervention pour l'application du dégagement mécanique. Ces résultats à moyen terme confirment ceux obtenus à plus court terme sur les stations riches de la sapinière (Thiffault et al. 2014b). D'une manière similaire, Fu et al. (2008) n'ont pas mesuré de différences significatives dans la taille de conifères plantés et soumis à des dégagements chimiques réalisés entre 1 et 5 ans après la mise en terre. Cette absence de différences peut être expliquée, à court terme, par l'importance du rôle de la compétition des herbacées lors des premières années de la plantation (Balandier et al. 2006; Fu et al. 2008). A plus long terme, l'absence d'effet marqué du délai d'application du dégagement mécanique suggère que la taille initiale des plants, laquelle était adaptée aux conditions de stations (dimensions plus élevées pour les stations les plus riches; Thiffault et Roy 2011), leur a conféré un potentiel de croissance permettant de surpasser la croissance rapide des rejets de souches des compétiteurs feuillus (Jobidon 1997; Jobidon et Charette 1997; Frey et al. 2003; Jobidon et al. 2003). De plus, l'absence d'interaction entre le scénario sylvicole et l'indice de compétition sur le diamètre des arbres - une variable très sensible à la compétition (Lanner 1985) - indique que l'environnement compétitif dans lequel ils ont évolué n'a pas affecté leur capacité à répondre à la compétition. Contrairement à notre prédiction, il semble que la compétition en bas âge nait pas influencé la morphologie des plants et leur biomasse foliaire avec une intensité telle qui aurait pu diminuer leur capacité à capter la lumière et ainsi modifier leur potentiel compétitif (Jobidon et al. 1998). Ces résultats confirment que le scénario combinant un reboisement hâtif à l'utilisation de plants d'une taille adaptée aux conditions de stations confère aux arbres plantés un avantage compétitif par rapport à la végétation concurrente (Jobidon et al. 1998; Lamhamedi et al. 1998; Thiffault et Roy 2011).

Nos résultats démontrent également l'importance du traitement de dégagement pour obtenir des plantations productives. En effet, nous montrons que pour des plantations d'épinettes blanche ou noire établies sur des stations riches de la sapinière, le nettoiement seul ne peut remplacer l'application d'un dégagement mécanique en bas âge. Le dégagement mécanique appliqué au moment requis résulte en une surface terrière des arbres plantés qui est de 64 à $105 \%$ supérieure à celle obtenue si le traitement est omis, et ce même avec l'application d'un nettoiement. La majorité des études à court (e.g., Thiffault et al. 2014b), moyen (e.g., Wagner et Robinson 2006), ou plus long termes (Cyr and Thiffault 2009) portant sur les gains en croissance associés à la gestion de la végétation concurrente démontrent des effets similaires (Wagner et al. 2006). Le dégagement est un traitement qui améliore la stabilité les tiges (Prégent 1998), comme le démontrent le plus faible ratio Hauteur/DHP des peuplements soumis au dégagement mécanique, lorsque comparé aux parcelles témoins. Ceci permet ainsi de diminuer l'âge auquel les peuplements pourront être soumis à une première éclaircie commerciale (Prégent 1998).

Notre étude démontre aussi que le dégagement mécanique a permis d'augmenter la surface terrière totale des peuplements mesurée entre 15 et 20 ans, quel que soit le site d'étude et sans égard au moment d'application du traitement. Le dégagement mécanique ou le nettoiement augmente généralement la surface terrière des essences désirées et diminue, en plus forte proportion, celle des essences non commerciales (Cyr and Thiffault 2009). Ainsi, l'accélération de la croissance des épinettes plantées soumises au dégagement mécanique a possiblement compensé la perte de rendement liée au retrait des espèces ligneuses non désirées. Le gain en rendement serait donc issu de l'application formelle de la stratégie de gestion de la végétation forestière préconisée au Québec (Thiffault and Roy 2011; Bureau du forestier en chef 2015) au sein de stations riches de la sapinière. Ensuite, le dégagement mécanique peut avoir favorisé la croissance des épinettes plantées au détriment d'espèces arbustives (telle Rubus idaeus L.) qui ne contribuent pas à la surface terrière, même si elles sont dominantes pendant les premières années de la succession. Au final, le dégagement mécanique permettrait d'exprimer une productivité forestière accrue sur certaines stations en libérant la régénération forestière de la pression compétitive des espèces concurrentes.

\section{Implications pour la sylviculture}

Nos résultats confirment que dans un scénario de reboisement hâtif avec des plants d'une taille adaptée comprenant également un traitement de nettoiement au stade gaulis, il n'y a pas d'impact sylvicole significatif à retarder l'application d'un dégagement mécanique jusquà deux années après le moment requis selon les normes opérationnelles au Québec. Cette marge de manœuvre opérationnelle devrait être utilisée afin d'assurer la réalisation des entretiens nécessaires. Au Québec, le Forestier en chef concluait en effet à un succès mitigé du dégagement mécanique dans la province, principalement en raison de lacunes associées aux suivis et à l'entretien des plantations (Bureau du forestier en chef 2015). L'enquête, réalisée dans cinq régions du Québec, faisait notamment ressortir que pour des raisons opérationnelles, plusieurs intervenants entretiennent les plantations davantage par le nettoiement seul (au stade gaulis) que par le dégagement (au stade semis) (Bureau du forestier en chef 2015). Or, nos résultats illustrent clairement l'effet négatif marqué d'omettre la réalisation du dégagement mécanique sur la production forestière dans les stations riches de la sapinière, tant à l'échelle des arbres qu'à celle du peuplement. En effet, la réalisation du dégagement 
mécanique permet d'augmenter la hauteur et le DHP des épinettes plantées et d'augmenter la surface terrière totale. Ainsi, nos travaux appuient le besoin d'effectuer des suivis et des entretiens adéquats des plantations en bas âge, afin qu'elles soient productives et présentent ainsi les rendements escomptés. Enfin, nous observons des différences de surface terrière totale du peuplement et des épinettes plantées entre les sites. Or, les effets de la végétation concurrente sur les plants varient selon les caractéristiques pédoclimatiques des sites ainsi que la nature et l'abondance des compétiteurs (Bell et al. 2000). Ces interactions sétendent aux traitements sylvicoles, dont les effets peuvent varier en fonction du climat, du sol, de la végétation concurrente et des types de plants mis en terre (Thiffault et al. 2014b). Il est donc crucial de prendre connaissances des interactions fines existant entre le traitement sylvicole et les caractéristiques des stations dans la prise de décision en sylviculture.

\section{Remerciements}

Nous remercions Jacques Carignan, Evelyne Gaillard, Réjean Poliquin, Maïté Brémont, Dominique Létourneau et Steve Lemay pour leur travail technique, lequel a permis l'établissement et le suivi des dispositifs expérimentaux. Nous remercions également le personnel des bureaux régionaux du ministère des Forêts, de la Faune et des Parcs du Québec (MFFP) pour leur collaboration soutenue, sans laquelle le suivi à long terme de ces dispositifs aurait été impossible. Nous soulignons la contribution de Maripierre Jalbert à l'édition des figures et de Josianne DeBlois aux analyses statistiques. Les commentaires constructifs de deux réviseurs anonymes et d'un éditeur associé ont permis d'améliorer une première version de ce manuscrit; nous les en remercions. Cette étude fait partie du projet 142332031 de la Direction de la recherche forestière du MFFP et a bénéficié d'un financement du Fonds de recherche Nature et Technologies du Québec (bourse postdoctorale à M. Urli).

\section{Références}

Balandier, P., C. Collet, J.H. Miller, P.E. Reynolds et S.M. Zedaker. 2006. Designing forest vegetation management strategies based on the mechanisms and dynamics of crop tree competition by neighbouring vegetation. Forestry 79(1): 3-27. doi:10.1093/forestry/cpi056. Base de données nationale sur les forêts. 2017. Sylviculture, 2015 [En ligne]. Disponible à http://nfdp.ccfm.org/silviculture/quick_ facts_f.php [page consultée le 25 octobre 2017].

Bell, F.W., M.T. Ter-Mikaelian et R.G. Wagner. 2000. Relative competitiveness of nine early-successional boreal forest species associated with planted jack pine and black spruce seedlings. Can. J. Forest Res. 30(5): 790-800. doi:10.1139/x00-004.

Biging, G.S. et M. Dobbertin. 1992. A comparison of distancedependent competition measures for height and basal area growth of individual conifer trees. For. Sci. 38(3): 695-720.

Bureau du forestier en chef. 2013. Manuel de détermination des possibilités forestières 2013-2018. Gouvernement du Québec, Roberval, QC.

Bureau du forestier en chef. 2015. Succès des plantations. Avis du Forestier en chef. FEC-AVIS-04-2015, Roberval, QC.

Cyr, G. et N. Thiffault. 2009. Long-term black spruce plantation growth and structure after release and juvenile cleaning: A 24-year study. Forest. Chron. 85(3): 417-426. doi:10.5558/tfc85417-3.

Frey, B.R., V.J. Lieffers, S.M. Landhäusser, P.G. Comeau et K.J. Greenway. 2003. An analysis of sucker regeneration of trembling aspen. Can. J. Forest Res. 33(7): 1169-1179. doi:10.1139/x03-053.
Fu, S., H.Y.H. Chen, F.W. Bell, M. Sharma, J.R. Delaney et G. Peterson. 2008. Effects of timing of glyphosate application on jack pine, black spruce, and white spruce plantations in northern Manitoba. Forest. Chron. 84(1): 37-45. doi:10.5558/tfc84037-1.

Gravel, J., N. Thiffault et F. Hébert. 2014. Le dégagement et le nettoiement mécaniques de peuplements résineux. Direction de l'aménagement et de l'environnement forestiers et Direction de la recherche forestière, Ministère des Forêts, de la Faune et des Parcs. DAEF2-067-F-01. Québec, QC.

Hoepting, M.K., R.G. Wagner, J. McLaughlin et D.G. Pitt. 2011. Timing and duration of herbaceous vegetation control in northern conifer plantations: 15th-year tree growth and soil nutrient effects. Forest. Chron. 87(3): 398-413. doi:10.5558/tfc2011-030.

Jobidon, R. 1992. Measurement of light transmission in young conifer plantations: A new technique for assessing herbicide efficacy. North. J. Appl. For. 9(3): 112-115.

Jobidon, R. 1994. Light threshold for optimal black spruce (Picea mariana) seedling growth and development under brush competition. Can. J. Forest Res. 24(8): 1629-1635. doi:10.1139/x94-211.

Jobidon, R. 1997. Stump height effects on sprouting of mountain maple, paper birch and pin cherry - 10 year results. Forest. Chron. 73(5): 590-595. doi:10.5558/tfc73590-5.

Jobidon, R. 2000. Density-dependent effects of northern hardwood competition on selected environmental resources and young white spruce (Picea glauca) plantation growth, mineral nutrition, and stand structural development - A 5-year study. Forest Ecol. Manag. 130(1-3): 77-97. doi:10.1016/S0378-1127(99)00176-0.

Jobidon, R. et L. Charette. 1997. Effets, après 10 ans, du dégagement manuel simple ou répété et de la période de coupe de la végétation de compétition sur la croissance de lépinette noire en plantation. Can. J. Forest Res. 27(12): 1979-1991. doi:10.1139/x97-166.

Jobidon, R., L. Charette et P.Y. Bernier. 1998. Initial size and competing vegetation effects on water stress and growth of Picea mariana (Mill.) BSP seedlings planted in three different environments. Forest Ecol. Manag. 103(2-3): 293-305. doi:10.1016/S03781127(97)00228-4.

Jobidon, R., V. Roy et G. Cyr. 2003. Net effect of competing vegetation on selected environmental conditions and performance of four spruce seedling stock sizes after eight years in Québec (Canada). Ann. For. Sci. 60(7): 691-699. doi:10.1051/forest:2003063.

Kuznetsova, A., P.B. Brockhoff et R.H.B. Christensen. 2016. lmerTest: Tests in linear mixed effects models. R Package. v 2.0.

Lamhamedi, M.S., P.Y. Bernier, C. Hébert et R. Jobidon. 1998. Physiological and growth responses of three sizes of containerized Picea mariana seedlings outplanted with and without vegetation control. Forest Ecol. Manag. 110(1-3): 13-23.

Lanner, R.M. 1985. On the insensitivity of height growth to spacing. Forest Ecol. Manag. 13(3-4): 143-148. doi:10.1016/0378-1127(85) 90030-1.

Littell, R.C., G.A. Milliken, W.W. Stroup, R.D. Wolfinger et O. Schabenberger. 2006. SAS System for Mixed Models, 2nd ed. SAS Institute Inc, Cary, NC.

Paquette, A. et C. Messier. 2010. The role of plantations in managing the world's forests in the Anthropocene. Front. Ecol. Environ. 8(1): 27-34. doi:10.1890/080116.

Park, A. et E.R. Wilson. 2007. Beautiful plantations: Can intensive silviculture help Canada to fulfill ecological and timber production objectives? Forest. Chron. 83(6): 825-839. doi:10.5558/ tfc83825-6.

Pitt, D.G., A.E. Morneault, P. Bunce et F.W. Bell. 2000. Five years of vegetation succession following vegetation management treatments in a jack pine ecosystem. North. J. Appl. For. 17(3): 100-109. Prégent, G. 1998. Léclaircie des plantations. Direction de la recherche forestière, Ministère des Ressources naturelles. Mémoire de recherche forestière. 133. Québec, QC.

Prégent, G., G. Picher et I. Auger. 2010. Tarif de cubage, tables de rendement et modèles de croissance pour les plantations dépinette 
blanche au Québec. Ministère des Ressources naturelles et de la Faune, Gouvernement du Québec. Mémoire de recherche forestière. 160. Québec, QC.

R Core Team. 2012. R: A language and environment for statistical computing. R Foundation for Statistical Computing, Vienna, Austria. Robitaille, A. et J.P. Saucier. 1998. Paysages régionaux du Québec méridional. Publications du Québec, Québec, QC.

Salmon, D. 2016. Ressources et industries forestières du Québec. Portrait statistique, édition 2016. Ministère des Forêts, de la Faune et des Parcs, Gouvernement du Québec. Québec, QC.

Saucier, J.P., A. Robitaille et P. Grondin. 2009. Cadre bioclimatique du Québec. Dans Manuel de foresterie, 2nd edition. Édité par Doucet, R. et M. Côté. Ordre des ingénieurs forestiers du Québec, Éditions Multimondes, Québec, QC. pp. 186-205.

Soil Classification Working Group. 1998. The Canadian System of Soil Classification. 3rd ed. Agriculture and Agri-Food Canada. Publication. 1646. Ottawa, ON.

Thiffault, N. et V. Roy. 2011. Living without herbicides in Québec (Canada): Historical context, current strategy, research and challenges in forest vegetation management. Eur. J. For. Res. 130(1): 117-133. doi:10.1007/s10342-010-0373-4.

Thiffault, N. et F. Hébert. 2013. Le dégagement et le nettoiement. Dans Le guide sylvicole du Québec. Tome 2. Les concepts et l'application de la sylviculture. Édité par Larouche, C., F. Guillemette, P. Raymond et J.-P. Saucier, Québec, QC. pp. 244-271.

Thiffault, N., R. Jobidon et A.D. Munson. 2014a. Comparing large containerized and bareroot conifer stock on sites of contrasting vegetation composition in a non-herbicide scenario. New Forest. 45(6): 875-891. doi:10.1007/s11056-014-9443-7.
Thiffault, N., F. Hébert, L. Charette et R. Jobidon. 2014b. Large spruce seedling responses to the interacting effects of vegetation zone, competing vegetation dominance and year of mechanical release. Forestry 87(1): 153-164. doi:10.1093/forestry/cpt048.

Wagner, R.G. et A.P. Robinson. 2006. Critical period of interspecific competition for four northern conifers: 10-year growth response and associated vegetation dynamics. Can. J. Forest Res. 36(10): 2474-2485. doi:10.1139/X06-058.

Wagner, R.G., K.M. Little, B. Richardson et K. McNabb. 2006. The role of vegetation management for enhancing productivity of the world's forests. Forestry 79(1): 57-79. doi:10.1093/forestry/cpi057.

Ward, C. et T. Erdle. 2015. Evaluation of forest management strategies based on Triad zoning. Forest. Chron. 91(1): 40-51. doi:10.5558/tfc2015-009.

Will, R.E., D. Markewitz, R.L. Hendrick, D.F. Meason, T.R. Crocker et B.E. Borders. 2006. Nitrogen and phosphorus dynamics for 13-year-old loblolly pine stands receiving complete competition control and annual $\mathrm{N}$ fertilizer. Forest Ecol. Manag. 227(12):155-168. doi:10.1016/j.foreco.2006.02.027.

Wyatt, S., M.H. Rousseau, S. Nadeau, N. Thiffault et L. Guay. 2011. Social concerns, risk and the acceptability of forest vegetation management alternatives: Insights for managers. Forest. Chron. 87(2): 274-289. doi:10.5558/tfc2011-014. 


\section{Annexe}

Tableau A1. Valeurs de la surface terrière des épinettes plantées des conifères et des feuillus régénérés naturellement et valeur de la surface terrière totale du peuplement $\left(\mathrm{m}^{2} \cdot \mathrm{ha}^{-1}\right.$ ) et leur proportion dans le peuplement $[\%]$ dans les peuplements non soumis à un dégagement mécanique [Témoin] et dans ceux soumis à un dégagement l'année requise [Requis], un an après l'année requise [Requis+1] ou deux ans après l'année requise [Requis+2] pour les trois sites d'étude : plantation d'épinette noire [Caseault] et plantations d'épinette blanche [Owen et Sainte-Perpétue]. Les données présentées sont les moyennes \pm l'écart-type $(n \leq 7$ blocs]. Les moyennes avec les mêmes lettres ne sont pas significativement différentes entre les traitements $[\alpha=0,05)$.

\begin{tabular}{|c|c|c|c|c|}
\hline & Témoin & Requis & Requis +1 & Requis +2 \\
\hline \multicolumn{5}{|l|}{ Caseault } \\
\hline \multicolumn{5}{|l|}{ Epinettes plantées } \\
\hline Surface terrière & $9,9 \pm 6,0 \mathrm{a}$ & $16,0 \pm 2,5 b$ & $18,2 \pm 2,6 b$ & $14,4 \pm 5,7 \mathrm{ab}$ \\
\hline Proportion & $67 \pm 21 \mathrm{a}$ & $90 \pm 10 \mathrm{~b}$ & $83 \pm 10 a b$ & $77 \pm 22 \mathrm{ab}$ \\
\hline \multicolumn{5}{|l|}{ Autres conifères } \\
\hline Surface terrière & $3,5 \pm 2,7 \mathrm{a}$ & $1,7 \pm 1,8 \mathrm{a}$ & $3,7 \pm 2,5 \mathrm{a}$ & $4,0 \pm 3,7 \mathrm{a}$ \\
\hline Proportion & $27 \pm 16 b$ & $9 \pm 10 \mathrm{a}$ & $17 \pm 10 \mathrm{ab}$ & $23 \pm 22 \mathrm{ab}$ \\
\hline \multicolumn{5}{|l|}{ Feuillus } \\
\hline Surface terrière & $0,3 \pm 0,5 \mathrm{a}$ & $0,0 \pm 0,1 \mathrm{a}$ & $0,0 \pm 0,0 \mathrm{a}$ & $0,0 \pm 0,0 \mathrm{a}$ \\
\hline Proportion & $7 \pm 11 \mathrm{a}$ & $0 \pm 0 \mathrm{a}$ & $0 \pm 0 \mathrm{a}$ & $0 \pm 0 \mathrm{a}$ \\
\hline \multicolumn{5}{|l|}{ Totale } \\
\hline Surface terrière & $13,8 \pm 6,4 \mathrm{a}$ & $17,8 \pm 2,4 \mathrm{ab}$ & $22,0 \pm 2,6 b$ & $18,5 \pm 3,6 \mathrm{ab}$ \\
\hline \multicolumn{5}{|l|}{ Owen } \\
\hline \multicolumn{5}{|l|}{ Epinettes plantées } \\
\hline Surface terrière & $8,6 \pm 1,8 \mathrm{a}$ & $15,2 \pm 2,9 b$ & $15,4 \pm 2,9 \mathrm{~b}$ & $15,8 \pm 2,2 b$ \\
\hline Proportion & $96 \pm 3 a$ & $98 \pm 3 \mathrm{a}$ & $98 \pm 2 \mathrm{a}$ & $99 \pm 2 \mathrm{a}$ \\
\hline \multicolumn{5}{|l|}{ Autres conifères } \\
\hline Surface terrière & $0,3 \pm 0,3 \mathrm{a}$ & $0,2 \pm 0,3 \mathrm{a}$ & $0,3 \pm 0,3 \mathrm{a}$ & $0,2 \pm 0,3 \mathrm{a}$ \\
\hline Proportion & $4 \pm 3 \mathrm{a}$ & $2 \pm 3 \mathrm{a}$ & $2 \pm 2 \mathrm{a}$ & $1 \pm 2 \mathrm{a}$ \\
\hline \multicolumn{5}{|l|}{ Feuillus } \\
\hline Surface terrière & $0,0 \pm 0,0 \mathrm{a}$ & $0,1 \pm 0,1 \mathrm{a}$ & $0,0 \pm 0,1 \mathrm{a}$ & $0,0 \pm 0,0 \mathrm{a}$ \\
\hline Proportion & $0 \pm 0 \mathrm{a}$ & $1 \pm 1 \mathrm{a}$ & $0 \pm 0 \mathrm{a}$ & $0 \pm 0 \mathrm{a}$ \\
\hline \multicolumn{5}{|l|}{ Totale } \\
\hline Surface terrière & $9,0 \pm 1,9 \mathrm{a}$ & $15,5 \pm 2,6 b$ & $15,8 \pm 2,9 \mathrm{~b}$ & $16,0 \pm 2,0 \mathrm{~b}$ \\
\hline \multicolumn{5}{|l|}{ Sainte-Perpétue } \\
\hline \multicolumn{5}{|l|}{ Epinettes plantées } \\
\hline Surface terrière & $6,6 \pm 0,7 \mathrm{a}$ & $13,9 \pm 2,8 b$ & $13,8 \pm 1,8 \mathrm{~b}$ & $12,8 \pm 5,8 b$ \\
\hline Proportion & $73 \pm 11 \mathrm{a}$ & $76 \pm 26 a$ & $92 \pm 9 \mathrm{a}$ & $79 \pm 31 \mathrm{a}$ \\
\hline \multicolumn{5}{|l|}{ Autres conifères } \\
\hline Surface terrière & $2,2 \pm 1,4 \mathrm{a}$ & $5,8 \pm 8,9 a$ & $1,2 \pm 1,5 \mathrm{a}$ & $3,2 \pm 5,0 \mathrm{a}$ \\
\hline Proportion & $24 \pm 12 \mathrm{a}$ & $23 \pm 27 a$ & $8 \pm 9 a$ & $21 \pm 31 \mathrm{a}$ \\
\hline \multicolumn{5}{|l|}{ Feuillus } \\
\hline Surface terrière & $0,3 \pm 0,2 \mathrm{~b}$ & $0,1 \pm 0,1 \mathrm{ab}$ & $0,1 \pm 0,1 \mathrm{ab}$ & $0,1 \pm 0,1 \mathrm{a}$ \\
\hline Proportion & $1 \pm 0 \mathrm{~b}$ & $1 \pm 0 \mathrm{ab}$ & $1 \pm 1 \mathrm{a}$ & $0 \pm 0 \mathrm{a}$ \\
\hline \multicolumn{5}{|l|}{ Totale } \\
\hline Surface terrière & $9,2 \pm 1,0 \mathrm{a}$ & $19,9 \pm 6,3 b$ & $15,2 \pm 2,0 \mathrm{ab}$ & $16,0 \pm 1,0 \mathrm{~b}$ \\
\hline
\end{tabular}

